\title{
Automated Fiber Placement Approach over Open-contoured Sculptured Surface
}

\author{
Shuyun Meng ${ }^{1, a^{*}}$, Xiulan Wen ${ }^{1, b}$, Hanzhong Liu, ${ }^{1, \mathrm{c}}$ and Youxiong $\mathrm{Xu}^{1, \mathrm{~d}}$ \\ ${ }^{1}$ School of Automation, Nanjing Institute of Technology, China

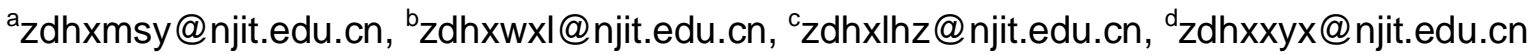

\begin{abstract}
Keywords: sculptured surface; fiber placement; a family of wraps
Abstract. By using the characteristic of tows and the core module surface, also including the demand of fiber placement, a family of wraps as fiber placement reference curves are presented based on equidistant calculation. A new algorithm for fiber placement of open contoured sculptured surface is proposed. Combined with the placement direction and the motion control parameter of the fiber placement head, a series of discrete placement points are obtained by projection using differential geometry theory. This algorithm can be applied in a variety of surface with equation description. The simulation shows that the algorithm is feasible and effective.
\end{abstract}

\section{Introduction}

Composite components are now widely used in aviation, aerospace, shipping industry. It is attractive for people due to being sparing in the use of raw materials, favorable weight loss ratio with more strength and stiffness. There is a trend that the traditional manual fiber placement method for complex composite components is replaced by automated fiber placement technology, which is now becoming more prevalent as one of additive manufacturing approaches.

Nowadays a number of scholars and researchers devote themselves to developing the methods of fiber placement path for composites. Schueler K. et al[1] provided a method that can approximate the individual tow path by means of repeatedly offsetting the initial curve on a free form surface. The error of approximation is analyzed and calculated due to the generation method of the series of fiber curves based on the same initial fiber path. Bijan,S.et al[2] presented that the fiber path can be generated via constructing the tag points which are the coordinate frames for position and orientation of the fiber placement head. If increasing the resolution, some new tag points can be inserted by the algorithm which carries out the weighted average between the two corresponding adjacent tag points. Wang X.et al[3]suggested a method that a series of uniform coverage curves are obtained with reference to an initial curve generated by solving the differential equations. Shao. et al[4] introduced an idea that can generate the trajectory for the meshing of unparallel borderline free-form surface. Wang. et al[5] proposed an algorithm which can design a locus with given ply orientation information for the free-form surface. Adriana W. Blom et al[6] persented the method that can derive the fiber placement path with a variable fiber angle for surface, which optimizes the course locations. Bijan Shirinzadeh et al[7] obtained the fiber placement trajectories by three methods, for example, calculating a series of tag points automatically for complex open surfaces or steering the fiber according to the predefined angle or sensory-based contour following methodology. Li. et al[8] presented a variable angle trajectory planning algorithm for two non-developable surfaces. Wen. et al[9] gived us a adjustment algorithm which diverts the placement direction from the origin one to the adjusted one,that is, the geodesic direction. Li. et al[10] presented a trajectory planning algorithm of automated fiber placement for meshed surface in fixed angle direction and analyzed the singular problem and non-solution problem. Shao. et al[11] studied the fiber placement path planning and optimization algorithm based on equivalent placement angle method and isometric offset method analysis. Huan. et al[12] proposed a trajectory generation algorithm which is with the fiber placement angle distribution function constructed by the least-squares method and the fiber orientations satisfying the fiber placement angle distribution function by the variable angle fiber placement. Lu. et al[13]offseted the initial paths continuously with the given placement angles in order to generate the fiber placement paths. Wang et al[14] researched the fiber placement trajectory planning method for 
the non-developable complex curve surface:the gradient angle change method and the arc transition method.

Different from the above mentioned, this paper presents a new approach of fiber placement for open contoured free form surface. The fiber placement paths are computed by defining a series of wraps as the initial reference curves and then generating the first path along the fiber placement direction with respect to the corresponding reference curve and finally offsetting the first path continuously with the width of tow.

\section{Generation a family of wraps as reference curves}

Now consider an open contoured free form surface, defined as $\mathbf{S}(\mathrm{u}, \mathrm{v})$, which is used to express the core module surface. The mathematical description of the surface $\mathbf{S}(\mathrm{u}, \mathrm{v})$ is given by Eq.(1)

$\mathbf{S}(\mathrm{u}, \mathrm{v})=\sum_{\mathrm{i}=0}^{\mathrm{m}} \sum_{\mathrm{j}=0}^{\mathrm{n}} \mathbf{D}_{\mathrm{i}, \mathrm{j}} \mathrm{N}_{\mathrm{i}, \mathrm{k}}(\mathrm{u}) \mathrm{N}_{\mathrm{j}, \mathrm{l}}(\mathrm{v})$

Where the set of $(m+1)$ rows and $(n+1)$ columns control points are $\mathbf{D}_{i, j}, i=0,1, \ldots, m, j=0,1, \ldots, n ; N_{i, k}(u)$ and $\mathrm{N}_{\mathrm{b}, \mathrm{k}}(\mathrm{v})$ are $\mathrm{k}$ degree and 1 degree $\mathrm{B}$-spline basis functions respectively, so it depends on the knot vectors by deboor iterative algorithms.

Suppose that one of the boundaries of the core module surface is the first wrap as an initial reference curve in the proposed algorithm. The other wraps can be derived by the following procedure. Now let the symbol $b$ represent the width of tow and the symbol $t$ indicate the number of the tow for a course. The first wrap is along the direction of parameter $\mathrm{v}$, so it can be expressed by the Eq.(2)

$\left.\mathbf{S}(u, v)\right|_{u=0}=0$.

The next adjacent wrap is parallel to the first wrap in the parameter domain, but it is sure that the distance between two adjacent wraps on the surface in the Cartesian coordinate system is constant. Due to the existing of the surface's curvature, the distance between these two neighboring wraps on the surface is not equidistant. Therefore, now consider a reference curves, that is, this is also an isoparametric curves. The start point of the curve, which is such a point corresponding to the zero value of parameter $\mathrm{v}$ on the isoparametric curves along parameter $\mathrm{u}$ direction, is snatched as an isometric point in order to facilitate the calculation. In that case, the equidistant calculation is performed continuously from the start point of the initial reference curve along the perpendicular direction of the tangent vector of fiber placement path on the surface. The isometric length on the surface is the width of tow every time.

To illustrate, consider the core module surface $\mathbf{S}(\mathrm{u}, \mathrm{v})$. If the tangent vector of the boundary curve at the starting point is expressed by symbol $\mathbf{T}$, then it can be calculated using Eq.(3).

$\mathbf{T}=\left.\left.\frac{d}{d v} \mathbf{S}(u, v)\right|_{u=c o n s t}\right|_{v=0}$.

where the value of const $=0,1$.

Supposed the fiber placement angle is given by symbol $\theta$, then the fiber placement direction vector $\mathbf{R}$ at this point can be evaluated by Eq.(4):

$\mathbf{R}=\mathbf{T} \times \mathbf{A}(\theta)$.

where $\mathbf{A}(\theta)$ is the rotational transform matrix with respect to the angle $\theta$, and $\mathbf{A}(\theta)$ can be calculated by Eq.(5) 


$$
\mathbf{A}(\theta)=\left[\begin{array}{cccc}
\cos \theta+(1-\cos \theta) n_{x}^{2} & (1-\cos \theta) n_{x} n_{y}-n_{z} \sin \theta & (1-\cos \theta) n_{x} n_{z}+n_{y} \sin \theta & 0 \\
(1-\cos \theta) n_{x} n_{y}+n_{z} \sin \theta & \cos \theta+(1-\cos \theta) n_{y}^{2} & (1-\cos \theta) n_{y} n_{z}-n_{x} \sin \theta & 0 \\
(1-\cos \theta) n_{x} n_{z}-n_{y} \sin \theta & (1-\cos \theta) n_{y} n_{z}+n_{x} \sin \theta & \cos \theta+(1-\cos \theta) n_{z}^{2} & 0 \\
0 & 0 & 0 & 1
\end{array}\right] .
$$

In Eq.(5) the rotational axis $\mathbf{N}\left(n_{x}, n_{y}, n_{z}\right)$ is the normal vector at the starting point on the reference curve, which is defined by Eq.(6).

$$
\mathbf{N}=\frac{\partial \mathbf{S}(u, v)}{\partial u} \times\left.\frac{\partial \mathbf{S}(u, v)}{\partial v}\right|_{u=u_{0}, v=0}
$$

So the equidistant offsetting direction is the vector $\mathbf{O}\left(\mathrm{o}_{\mathrm{x}}, \mathrm{o}_{\mathrm{y}}, \mathrm{o}_{\mathrm{z}}\right)$ which is calculated by Eq.(7). $\mathbf{O}=\mathbf{R} \times \mathbf{N}$.

Now construct the first plane that is perpendicular to vector $\mathbf{O}$ and pass through the first point of the initial reference curve in Fig. 1. Then the first plane is offset consequently for $t$ times. The offset distance is the width of the tow every time. Finally the last offset plane, which is parallel to the first plane, will intersect with the surface $\mathbf{S}$. In that way, intersection points which lie on the border of the surface $\mathbf{S}$ can be derived by solving the following equations.

Suppose that a point $\mathrm{B}\left(\mathrm{b}_{\mathrm{x}}, \mathrm{b}_{\mathrm{y}}, \mathrm{b}_{\mathrm{z}}\right)$ on the first plane. After offsetting along the vector $\mathbf{O}$ for $\mathrm{t}$ times, the new coordination of point $\mathrm{B}$ is achieved by Eq.(8)

$\mathbf{B}_{1}=\mathbf{B}_{0}+b^{*} t * \mathbf{O}$.

So the formula of the last offset plane is expressed by Eq.(9)

$$
\mathbf{O} \cdot\left(\mathbf{r}-\mathbf{B}_{1}\right)=0 \text {. }
$$

Then the preceding intersection point of the last offset plane and the module surface is calculated by solving the Eq.(1) and (9) with the constraint condition, that is, let the value of parameter $\mathrm{v}$ be equal zero.In other words, this point is such one which locates on the $u$ parametric line of the fiber placement surface $\mathrm{S}$. Thus the second reference line is the u parametric line which goes through this intersection point. Of course, the rest of reference lines can be obtained by this method, so a family of wraps as reference lines are now generated.

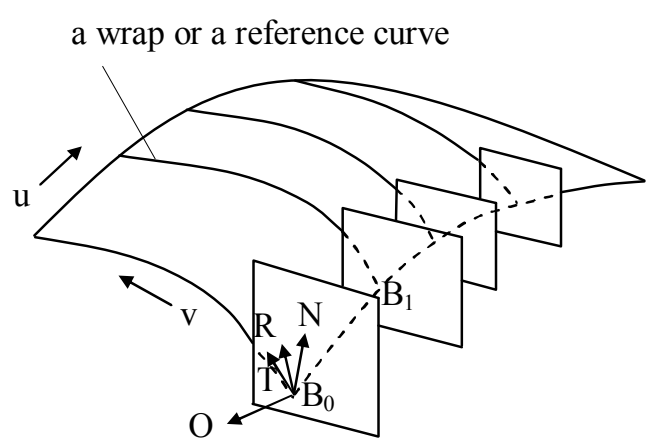

Fig. 1 the generation of a family of wraps

\section{Geneation of fiber placement points for open-contoured free form surface}

Fiber placement paths are the continuous curves on the core module surface, which are presented as the curvilinear mathematical equations or a series of discrete points sets. Here choose the latter one due to the difficulty of directly obtaining the explicit equation of the entire fiber path curve.

As stated earlier, there are two steps to implement in order to gain the fiber placement points. The first step is to confirm the fiber placement direction; the second is to determine the pace of fiber placement. The family of wraps is used as a reference curves when confirming the fiber placement direction. All of the paths which lie in the area between the neighboring two wraps can be generated 
by selecting one of the two wraps as a reference curve, and every fiber placement point on this path correlates with the the corresponding reference curve.

In the preceding section, the family of wraps as reference curves can be expressed by Eq.(10)

$\mathbf{S}(u, v)=0, u \in\left[u_{1}, u_{2}, \mathrm{~L}, u_{q}\right]$.

where the parameter $v$ is a variable, and $u_{1}, u_{2}, \ldots, u_{\mathrm{q}}$ are constant. The first point on the first tow for a course is already obtained by adopting the equidistant strategy in the preceding section. The direction in which the next point lies on the first tow can be calculated by Eq.(4). As shown in Fig.2, firstly to extend a predefined distance $\mathrm{L}$ along the fiber placement direction from the first point $\mathrm{C}$ to the new point $C^{\prime}$; secondly to project the point $C^{\prime}$ on the module surface $\Omega$, and then the projection point $C 1$ is a new fiber placement point.

The distance $\mathrm{L}$ is approximately computed with the technique of differential geometry. From the view point of kinematics and motion control, the distance is related with the interpolation velocity and the interpolation time; that is, the distance is the velocity multiplied by the time. The rest fiber placement points on the same tow can be attained using the same method.

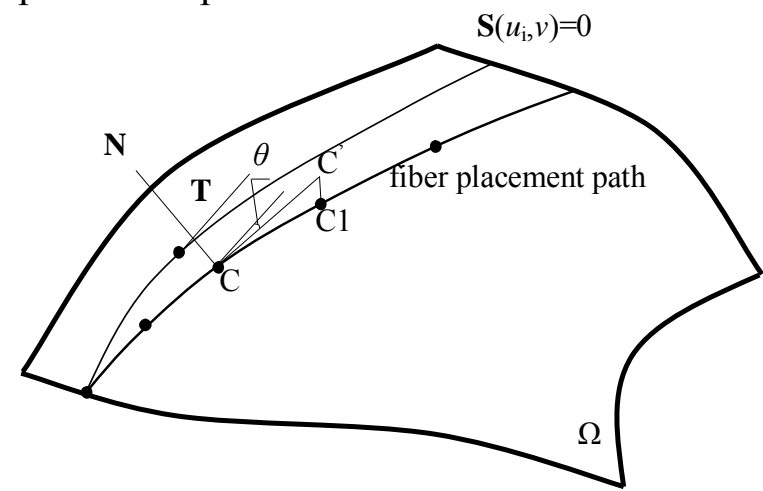

Fig.2 Generation of the first fiber path

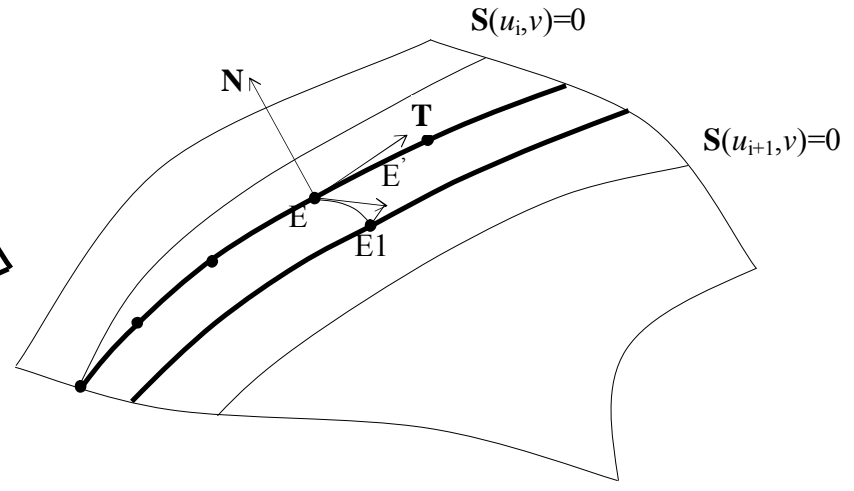

Fig. 3 Generation of the rest fiber paths

Generation of the paths of the rest tows for a course need to equidistant calculation from the corresponding former path. Of course, two steps are implemented just like the aforementioned: at first, to determine the direction; then to impose the equidistant calculation and to project this point to the module surface. The difference is that the calculation of direction and distance. As shown in Fig.3, the vector $\mathbf{T}$ is the tangent one at the fiber placement point on the former path, and the vector $\mathbf{N}$ is the normal one at this point on the module surface. The vector $\mathrm{EE}^{\mathrm{w}}$ representing the fiber placement direction is perpendicular to these two vectors $\mathbf{N}$ and $\mathbf{T}$ and can be estimated using the Eq.(11)

$\mathrm{EE}^{\prime} \times(\mathbf{N} \times \mathbf{T})=0$

Where $|E E|=b$, in other words, the offsetting distance is the width $\mathrm{b}$ of the single tow. After obtaining the offsetting point E', this point E' should be projected on the surface. The projection point E1 is new fiber placement one lying on the current path. All the points can be computed using the method until reaching the border of the module surface.

\section{Numerical Simulation Examples}

Here the proposed algorithm can be applied to these surfaces which can be expressed by mathematical equations, such as implicit or explicit surface, but not the meshing surfaces. Now consider a B-spline surface as the core module surface to verify the proposed method correctness.

Supposed that the tow-width is $1 / 4$ inch. There are 5 tows in a course. The angle is 45 degree between the fiber placement path and the corresponding wrap as a reference curve. The velocity of fiber placement head in the motion control system is $25 \mathrm{~m} / \mathrm{min}$ and the control period of the control system is $0.005 \mathrm{~ms}$. Fig. 4 and Fig. 5 individually shows the family of wraps as reference curves and the fiber placement path. The simulation is implemented in the platform MATLAB. 


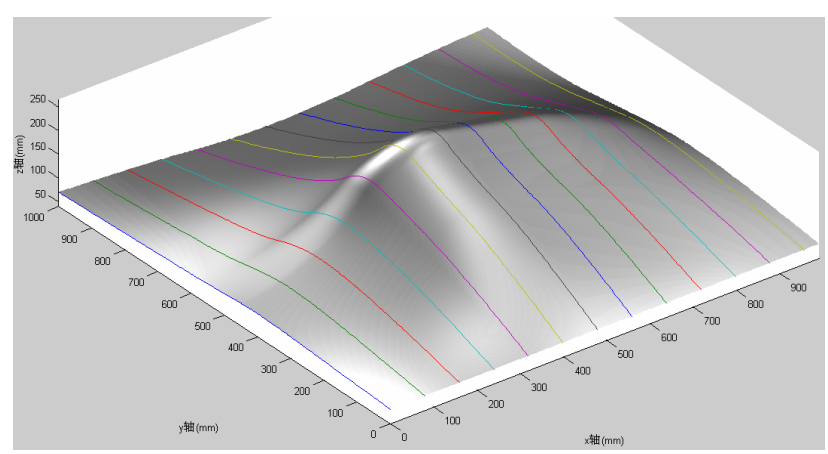

Fig.4 A family of wraps as reference curves

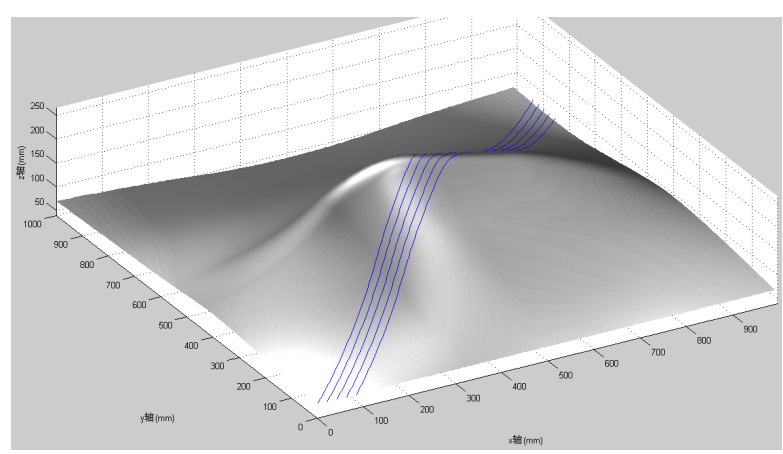

Fig.5 Generation of tow paths for a course

\section{Conclusions}

Comparing to the traditional method, this study has presented a novel approach about the path generation of fiber placement for open contoured sculptured surface. The method can be divided into two steps to be implemented. First, to generate a family of wraps based on equidistant calculation along the border of the surface; Second, according to the fiber placement direction and isometric distance, the first path and the rest of tows for a course individually are generated. Every point can be allowed to calculate at a arbitrary predefined angle that is relative to the corresponding wrap. This method is practically easy to realize. Further search will focus on the fiber placement of like rotary shell, such as fairing, air inlet and so on.

\section{References}

[1]Kurt Schueler, James Miller and Richard Hale. (2004). Journal of Computing and Information Science in Engineering, vol. 4 No.9, pp.251-256.

[2]Bijan Shirinzadeh, Chee Wei Foong and Boon Hui Tan. (2000). Assembly Automation, vol.20, No.4, pp.313-320.

[3]Xiaoping Wang, Luling An, Liyan Zhang and Laishui Zhou (2008).Chinese Journal of Aeronautics, vol.21,571-577.

[4]Z.X. Shao, H.Y. Fu, D.C. Li(2009). Key Engineering Materials, vol.392-394,pp.682-687.

[5]Peiyuan Wang, Yong Li, Xianfeng Wang and Jun Xiao(2011).Polymers\&Polymer Composites, vol.19,No.2\&3, pp.203-208.

[6]Adriana W. Blom, Mostafa M. Abdalla, Zafer Gurdal(2010).Composites Science and Technology, vol.70,pp.564-570.

[7]Bijan Shirinzadeh, Gursel Alici, Chee Wei foong, Gary Cassidy(2004).Robotics and Computer-Integrated Manufacturing, vol.20, pp.17-28.

[8]Yuehua Li, Hongya Fu, Zhenyu Han, Dedong Han(2013).Jisuanji Fuzhu Sheji Yu Tuxingxue Xuebao, vol.25,pp.1523-1529.

[9]Liwei Wen,Junfei Li, Xianfeng Wang, Jun Xiao (2013).Hangkong Xuebao, vol.34,pp.1731-1739. [10]Junfei Li, Xianfeng Wang, Jun Xiao, Wenlei Xiong (2013).Jisuanji Fuzhu Sheji Yu Tuxingxue Xuebao, vol.25,pp.1523-1529.vol.25,pp.1410-1415.

[11]Zhongxi Shao, Hongya Fu, Zhenyu Han, Yuan Liu (2010). Yuhang Xuebao,vol.31,pp.855-861.

[12]Dajun Huan, Jun Xiao, Yong Li (2011).Nanjing Li Gong Daxue Xuebao,vol.35,pp.410-414.

[13]Min Lu, Laishui Zhou, Luling An, Zhiguo Wang(2011).Nanjing Hangkong Hangtian Daxue Xuebao,vol.42,pp.735-738.

[14]Xianfeng Wang, Liwei Wen, Jun Xiao(2010).Journal of Advanced Materials,vol.42,pp.56-61. 